\title{
Pancreatitis-associated unique duodenal fistula: an autopsy
}

\section{case}

\author{
Sho Ogata ${ }^{*}$ and Ben Hatano ${ }^{1,2,3}$ \\ ${ }^{1}$ Department of Pathology and Laboratory Medicine, National Defense Medical College, Tokorozawa, Saitama, Japan \\ ${ }^{2}$ Department of Laboratory Medicine, National Defense Medical College, Tokorozawa, Saitama, Japan \\ ${ }_{3}^{3} J a p a n$ Development, Hematology Clinical Development Department, Novartis Pharma KK, Minato, Tokyo, Japan
}

\begin{abstract}
Duodenal fistula may be caused by severe pancreatitis, although viral pancreatitis is usually mild. We present a unique duodenal fistula that occurred upon repeated (fatal) myocardial infarctions during viral pancreatitis. Autopsy revealed a duodenal fistula with a unique appearance: it had two orifices, the proximal of which involved the minor papilla, and two intrapancreatic extensions (one along the course of the Santorinii duct and one along a so-called fusion line of pancreatic anlages). Three conditions superimposed upon cytomegalic viral pancreatitis [1) Santorinii duct obstruction, 2) athero-embolism, and 3) a so-called fusion line] may have contributed to this unique fistula formation.
\end{abstract}

\section{Introduction}

Gastrointestinal fistula is a well-known complication of acute necrotizing pancreatitis, and common sites for such pancreatitisassociated fistulas are the colon, jejunum, and duodenum. Duodenal fistula has been observed in $0-24 \%$ of severe pancreatitis, with most being located in its descending portion [1,2]. Cytomegalovirus (CMV) frequently produces only mild pancreatitis, which is not usually clinically apparent [3]. Fat necrosis or duct necrosis seems not to accompany this type of pancreatitis [4]. We present here an autopsy case manifesting a unique duodenal necrotic fistula that occurred during CMV pancreatitis and discuss the mechanisms that may have led to its formation.

\section{Report of a case}

A 76-year-old, diabetic, Japanese man was admitted to the National Defense Medical College Hospital (Tokorozawa, Japan) because of a recent myocardial infarction and renal failure. He had past histories of diabetic retinopathy, cholelithiasis, and brain and myocardial infarctions. On admission this time, he was given supportive care for cardiovascular collapse. He was also given continuous hemodialysis for intractable oliguria. His vital signs became stable during his hospitalization, but his serum amylase (p-isozyme-dominant) values rose gradually to 1,019 $\mathrm{IU} / \mathrm{ml}$, and serum lipase rose to $654 \mathrm{IU} / \mathrm{ml}$. An abdominal echogram and a computed tomogram (Figure 1A) each showed enlargement of the pancreas head. These findings were consistent with acute pancreatitis. Following intravenous administration of proteinase inhibitors, the serum values of the above enzymes returned to within normal limits. However, he experienced repeated heart attacks, and died a month after his pancreatitic event.

\section{Autopsy findings}

In the second portion of the duodenum, about $4 \mathrm{~cm}$ proximal to the major duodenal papilla, a fistula, unique in having two duodenal orifices, was found (Figure 1B, C). The proximal orifice involved the minor duodenal papilla, while the distal one was located between the two duodenal papillae. From these orifices, a wide necrotic cavity was located in the duodenal wall, with loss of the proper muscle layer, and it extended into the pancreas at two sites. The proximal of these two extensions involved the site of the Santorinii pancreatic duct, which contained stone-like concretions (Figure 1D) and fragments of hyperplastic duct epithelia. These suggested chronically obstructive changes in the Santorinii duct. The other extension passed along a socalled fusion line of pancreatic anlages. The fistula was situated away from the Wirsung pancreatic duct, choledochus, and a concomitant juxta-papillary duodenal diverticulum. The background pancreatic parenchyma displayed edema, lymphocytic infiltration, and viral inclusion bodies, findings consistent with CMV pancreatitis (Figure $1 \mathrm{E}, \mathrm{F})$. In addition, organizing cholesterol emboli were revealed within small branching blood vessels (Figure 1G). The duodenal wall exhibited submucosal fibrosis and hemosiderin deposition in a widely distributed fashion, findings considered to reflect a chronic ischemic condition.

\section{Comment}

The pathogenesis of pancreatitis-associated gastrointestinal fistulas is considered to be multifactorial, and to include activation of intrinsic pancreatic enzymes and local ischemia [1]. A pancreatic pseudocyst may communicate with the duodenal lumen in severe necrotizing pancreatitis [5]. It is well-known that CMV pancreatitis is usually not accompanied by tissue necrosis. In the present case, however, three unfavorable conditions seemed to be superimposed upon it: 1) necrosis of the Santorinii duct (and its orifice; i.e., the minor duodenal papilla) due to obstructive changes, 2) local hypoperfusion due to

Correspondence to: Sho Ogata, Department of Pathology and Laboratory Medicine, National Defense Medical College, 3-2 Namiki, Tokorozawa, Saitama, 359-8513 Japan, Tel: +81-4-2995-1505; Fax: +81-4-2996-5192; E-mail: sogata@ ndmc.ac.jp

Key words: cytomegalovirus, duodenal fistula, pancreatitis, fusion-line, cholesterol embolism

Received: April 06, 2018; Accepted: April 23, 2018; Published: April 27, 2018 


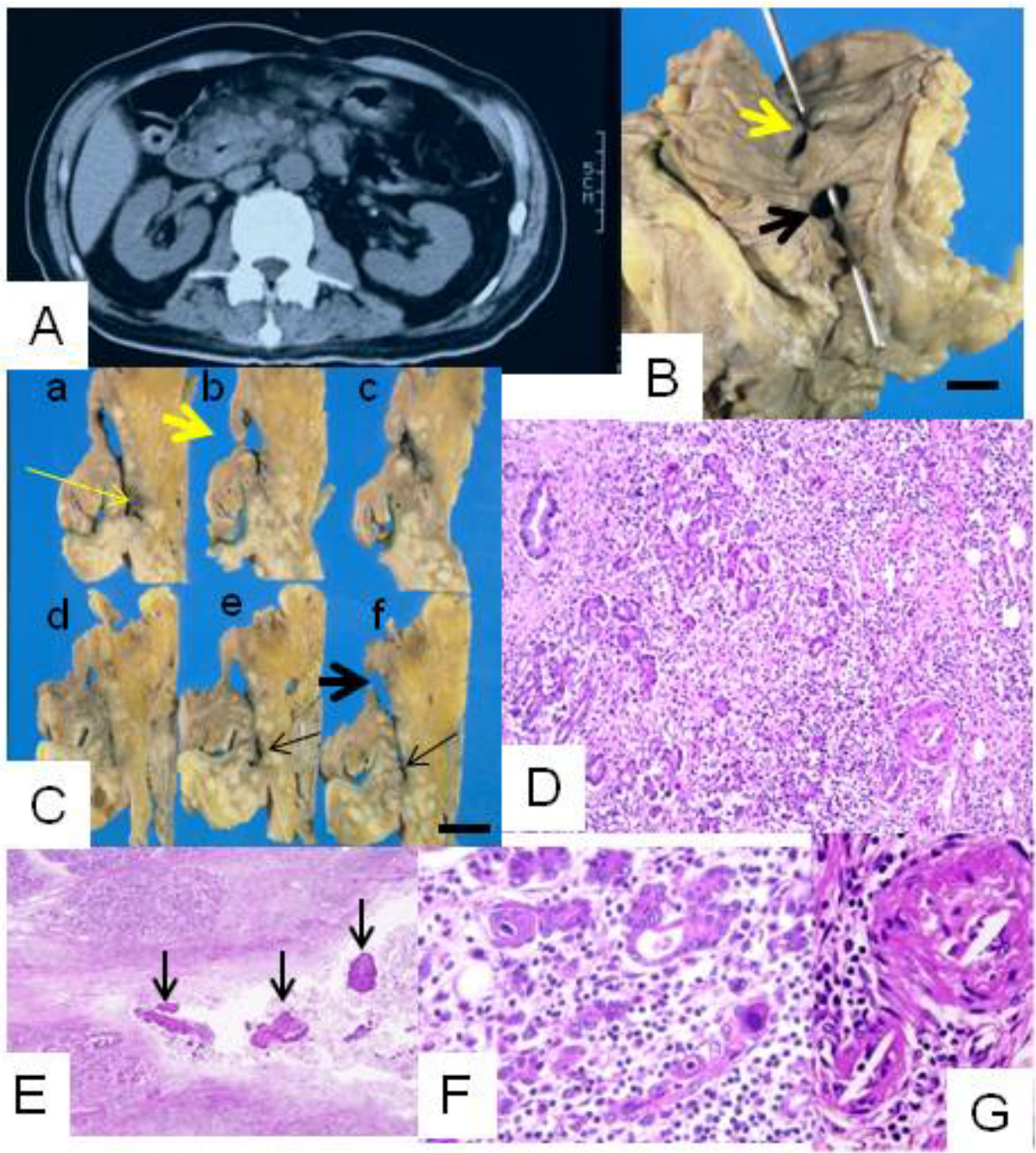

Figure 1A. Abdominal computed tomography revealed swelling of pancreas head. A white arrow indicated air-filled space within pancreas head.

B. Gross appearance of the unique duodenal fistula. It communicated with the duodenal lumen via two orifices [a probe is shown projecting from the proximal orifice (thick yellow arrow) and passing via a necrotic cavity to the distal orifice (thick black arrow)]. Indicated scale, $1 \mathrm{~cm}$

C. Serially cut surfaces of the duodenopancreas region from cranial (a) to caudal (f). Proximal and distal orifices, and the proximal and distal intrapancreatic extensions of this fistula are indicated by thick yellow and black arrows and thin yellow and black arrows, respectively. Indicated scale, $1 \mathrm{~cm}$.

D. Proximal intrapancreatic extension of the fistula contained concretions (arrows; H\&E; x40).

E. Edema and marked lymphocytic infiltration were observed (H\&E; $\mathrm{x} 100)$.

F. Some epithelial cells had cytomegalic viral inclusion bodies (H\&E; x200)

G. Arterioles were obstructed and contained needle-like structures, suggesting cholesterol embolism (H\&E; x200).

athero-embolism, and 3) structural fragility of a so-called fusion line of pancreatic anlages.

Concerning the first unfavorable condition, obstruction of the pancreatic duct is considered one of the main mechanisms associated with acute pancreatitis [6]. In the present case, the Santorinii duct itself caused necroinflammation (forming both the proximal orifice and the proximal extension of the fistula), and this inflammation may have propagated directly to the duodenal wall (forming the wide cavity seen in the duodenal wall). Local ischemia caused by athero-embolism, the second unfavorable condition, may have caused secondary structural fragility and exacerbated the degree of inflammation [7]. This factor may have contributed to the formation of the cavity in the duodenal wall and the distal orifice. The last unfavorable condition is a native structural fragility in the pancreas. A so-called fusion-line of pancreatic anlages tends to be subject to involvement in inflammation [8]. In our case, the distal intrapancreatic extension probably have resulted from inflammation propagated along this anatomically fragile site. In this situation, the role of the viral pancreatitis itself in the formation of the unique fistula is not clear, although it may have exacerbated the necroinflammation, such as that secondary to the local ischemia resulting from athero-embolism.

In conclusion, we present a unique duodenal fistula that accompanied CMV pancreatitis. We considered necrosis of the Santorinii pancreatic duct to be the best candidate for the main mechanism, and the inflammation to have been exacerbated by two conditions that lead to structural fragility: hypoperfusion due to atheroembolism and the presence of a so-called fusion line of pancreatic anlages. Viral pancreatitis may have contributed by its exacerbating those conditions. 


\section{References}

1. Sarr MG (1998) Gastrointestinal fistulas complicating surgical management of necrotizing pancreatitis. In: Beger $\mathrm{HG}$, Warshaw AL, Buechler MW, et al., eds. The pancreas. Oxford, Blackwell Science 594-598.

2. Doberneck R (1989) Intestinal fistula complicating necrotizing pancreatitis. Am J Surg 158: 581-584. [Crossref]

3. Cappell MS, Hassan T (1993) Pancreatic disease in AIDS--a review. J Clin Gastroenterol 17: 254-263. [Crossref]

4. Kloeppel G, Maillet B (1998) Histopathology of acute pancreatitis. In: Beger HG, Warshaw AL, Buechler MW, et al., eds. The pancreas. Oxford, Blackwell Science 404409.
5. Arifuddin R, Baichi M, Ullah A (2007) Pancreatic pseudocysts and a cyst-duodenal fistula complicating severe necrotizing pancreatitis from pancreas divism. Dig Dis Sci 52: 3143-3144. [Crossref]

6. Lerch MM, Saluja AK, Dawra R, Ramarao P, Saluja M, et al. (1993) Pancreatic duct obstruction triggers acute necrotizing pancreatitis in the opossum. Gastroenterology 104: 853-861. [Crossref]

7. Matsukuma S, Suda K, Abe H (1998) Histological study of pancreatic ischemic lesions induced by cholesterol emboli: fresh and subsequent features of pancreatic ischemia. Human Pathol 29: 41-46. [Crossref]

8. Suda K, Mizuguchi K, Matsumoto M (1983) A histopathological study on the etiology of duodenal diverticulum related to the fusion of the pancreatic anlage. $\mathrm{Am} \mathrm{J}$ Gastroenterol 78: 335-338. [Crossref]

Copyright: (C2018 Ogata S. This is an open-access article distributed under the terms of the Creative Commons Attribution License, which permits unrestricted use, distribution, and reproduction in any medium, provided the original author and source are credited. 